\title{
Europäische Lohnkoordinierung: solidarisch oder wettbewerbsorientiert?
}

\author{
THORSTEN SCHULTEN
}

Vor dem Hintergrund der aktuellen Euro-Krise hat die Frage nach Möglichkeiten und Grenzen einer europäischen Koordinierung der Lohnpolitik wieder deutlich an Aktualität gewonnen. In den vorherrschenden Krisendiagnosen wird der Lohnfrage zumeist ein zentraler Stellenwert eingeräumt. Im Mittelpunkt steht dabei die weit verbreitete These, wonach die zunehmenden ökonomischen Ungleichgewichte in Europa zwischen den sogenannten „Defizit-“ und den sogenannten „Überschussstaaten“ vor allem das Ergebnis einer stark auseinanderlaufenden Lohn- und Lohnstückkostenentwicklung sind. Letztere wird als zentrale Ursache für die unterschiedlichen Inflationsraten in Europa gesehen, die zu einer erheblichen Verschiebung der (preislichen) Wettbewerbsfähigkeit geführt hätten. Den Defizitstaaten im Süden Europas wird dabei unterstellt, durch vermeintlich zu hohe Lohnabschlüsse in den 2000er Jahren ihre Wettbewerbsfähigkeit ruiniert und dadurch immer höhere Leistungsbilanzdefizite produziert zu haben. Demgegenüber gelten die Überschussstaaten im Norden Europas oft als vorbildlich, weil hier eine vergleichsweise moderate Lohnentwicklung zu hoher Wettbewerbsfähigkeit und damit verbunden steigenden Exportüberschüssen geführt habe.

Gegenüber dieser „asymmetrischen“ Betrachtungsweise sehen andere hingegen auch die Lohnentwicklung im Norden eher kritisch, weil sie durch „zu niedrige “ Lohnabschlüsse ihrerseits die wirtschaftlichen Ungleichgewichte in Europa verschärft hat. Insbesondere Deutschland sieht sich hierbei dem Vorwurf ausgesetzt, seine Exportstärke durch eine aktive Lohndumpingstrategie befördert zu haben. Diese sei umso schwerwiegender, weil innerhalb der Euro-Zone Unterschiede in der nationalen Wettbewerbsfähigkeit nicht mehr durch eine entsprechende Aufoder Abwertung der nationalen Währungen ausgeglichen werden könnten.

Folgt man dieser herrschenden Sicht auf die Lohnpolitik, dann gibt es für den Abbau wirt- schaftlicher Ungleichgewichte in Europa lediglich zwei Lösungen, die mit der Alternative interne oder externe Abwertung auf den Begriff gebracht werden. Interne Abwertung bedeutet, dass die Defizitländer versuchen, durch massive Kürzungen von Lohn- und Arbeitskosten ihre verlorene Wettbewerbsfähigkeit wiederherzustellen. Bei einer „symmetrischen“ Betrachtungsweise müssten respektive auch die Überschussländer eine Politik der internen Aufwertung verfolgen und durch eine expansive Lohnpolitik ihre „zu hohe“ Wettbewerbsfähigkeit abbauen. Hält man hingegen den Weg der internen Ab- und Aufwertung für politisch weder gangbar noch wünschenswert, so bleibt innerhalb dieser Logik nur die externe Auf- und Abwertung. Dies wäre jedoch nur bei einer Auflösung der Europäischen Währungsunion und einer Rückkehr zu nationalen Währungen möglich, wie sie mittlerweile zum Beispiel von führenden Autoren des Kölner Max-Planck-Institutes für Gesellschaftsforschung nahegelegt wird (s. a. den Beitrag von Höpner in diesem Heft).

\section{Ein alternativer Blick auf die euro- päische Lohnentwicklung}

Die vorherrschende Sichtweise auf die Lohnpolitik in Europa zeichnet sich sowohl in ihrer "asymmetrischen“ als auch in ihrer „symmetrischen" Variante dadurch aus, das sie die Löhne in erster Line als Kostenfaktor betrachtet und diese zur zentralen Variable nationaler Wettbewerbsfähigkeit erhebt. Gegen eine solche Sichtweise lassen sich mindestens drei grundlegende Einwände formulieren: Erstens bewegt sie sich auf einer rein makroökonomischen Ebene, die vollständig von den realwirtschaftlichen Strukturen der einzelnen EU-Staaten abstrahiert. Dabei geraten jedoch neben den preislichen auch die nicht-preislichen Faktoren der Wettbewerbsfähigkeit vollkommen aus dem Blickfeld. Hierbei geht es vor allem um das jeweilige sektorale Spezialisierungsprofil einer Volkswirtschaft sowie um die nationale Wirtschaftskultur im Hinblick auf die Qualität und Originalität bestimmter Produkte und Dienstleistungen. Schaut man z. B. auf die Struktur der deutschen Exportindustrie, so finden sich dort vielen Branchen (vor allem Maschinenbau, Chemie, Automobilindustrie usw.), bei denen nicht-preisliche Wettbewerbskomponenten entscheidend sind und Löhne (selbst unter Berücksichtigung der Vorleistungen) nur noch eine untergeordnete Rolle spielen. Umgekehrt führen selbst drastische Lohnsenkungen (wie z.B. aktuell in Griechenland) keineswegs automatisch zu mehr Wettbewerbsfähigkeit, wenn entsprechende realwirtschaftliche Strukturen fehlen. Damit wird jedoch der Stellenwert der Löhne für die Erklärung ökonomischer Ungleichgewichte in Europa stark relativiert.

Der zweite Einwand bezieht sich auf die Bedeutung der Löhne für die Preisentwicklung. Nach der herrschenden Sichtweise werden die Preise im Wesentlichen durch die nominalen Lohnstückkosten bestimmt. In der Tat lässt sich empirisch oft eine relativ ähnliche Entwicklung von Preisen und Lohnstückkosten feststellen. Allerdings sagt dies noch nichts über den tatsächlichen Wirkungszusammenhang beider Variablen. So können Preise auch durch eine Vielzahl von anderen Faktoren bestimmt werden. In Spanien waren es z. B. vor allem der Immobilienboom plus die stark ansteigenden Rohstoff- und Energiekosten, die in den 2000er Jahren zu einer besonders großen Preisdynamik geführt haben. Die Löhne haben demgegenüber im Wesentlichen die nationale Preisentwicklung nachvollzogen. Dabei waren die spanischen Gewerkschaften vor allem darauf bedacht, durch die Vereinbarung von Indexierungsklauseln in Tarifverträgen das Reallohnniveau der Beschäftigten zu sichern.

Der dritte Einwand gegen die herrschende Sichtweise auf die Lohnpolitik besteht schließlich darin, dass der eigentliche Verteilungskonflikt, d.h. die Auswirkungen der Lohnentwicklung für die Einkommensverteilung zwischen 
Kapital und Arbeit, vollkommen ausgeblendet wird. Damit wird zugleich die Bedeutung der Löhne als wesentlicher Faktor der gesamtwirtschaftlichen Nachfrage negiert. Betrachtet man jedoch nicht nur die nominale, sondern auch die reale Entwicklung der Lohnstückkosten in Europa, so fällt auf, dass nicht nur im Norden, sondern auch in den meisten südeuropäischen Ländern die Reallohnentwicklung hinter der Produktivität zurückgeblieben ist. Nahezu überall in Europa zeigt sich eine gleichförmige Entwicklung sinkender Lohnquoten, die sich lediglich im Tempo, nicht jedoch in der Richtung unterscheidet.

Mit der anhaltenden Umverteilung von Arbeits- zu Kapitaleinkommen geht jedoch eine strukturelle Nachfrageschwäche einher, auf die in den 2000er Jahren auf unterschiedliche Weise reagiert wurde. Während die Defizitstaaten die einkommensbedingten Nachfrageausfälle durch eine immer größere private Verschuldung kompensierten, wurde in den Überschussstaaten der schwachen Binnenkonjunktur durch eine immer stärkere Fixierung auf die Exportindustrie entgegengetreten. Sowohl das schuldengetriebene Wachstumsmodell des Südens als auch das exportgetriebene Wachstumsmodell des Nordens bedingen sich dabei gegenseitig und haben $\mathrm{zu}$ immer größeren ökonomischen Ungleichgewichten geführt. Mit dem Ausbruch der Krise wurde schließlich deutlich, dass beide Wachstumsmodelle keine nachhaltige Perspektive für Europa bieten.

\section{Die faktisch bestehende Lohn- koordinierung in Europa}

Angesichts des mittlerweile erreichten ökonomischen und politischen Integrationsgrades in Europa lautet die Kernfrage heute nicht mehr, ob eine europäische Lohnkoordinierung möglich ist, sondern welche Richtung der europäischen Lohnkoordinierung sich mit welchen dominierenden Akteuren und Institutionen faktisch durchsetzt. Die Geschichte der europäischen Lohnkoordinierung lässt sich dabei bis in die 1980er Jahre zurückverfolgen. Die Einführung des Europäischen Binnenmarktes hat schon damals zu einer erheblichen Intensivierung des Wettbewerbs in Europa geführt, in dessen Folge das bis heute dominierende Muster sinkender Lohnquoten entstanden ist. Die Koordinierung der Löhne erfolgte über den Markt und wurde durch umfangreiche Liberalisierungspolitiken vorangetrieben. Die Einschätzung, dass der Wettbewerbsdruck auf die
Löhne damals noch durch mögliche Wechselkursanpassungen abgefedert wurde, gehört zu den gut gepflegten Mythen derjenigen, die heute die vermeintlich "gute alte Zeit“ vor Einführung des Euro beschwören. Gerade in Deutschland wurden Aufwertungen der D-Mark von den Unternehmen stets zum Anlass genommen, um von den Gewerkschaften als Kompensation für den Verlust preislicher Wettbewerbsfähig keit Lohnzurückhaltung einzufordern.

In den 1990er Jahren hat sich dann mit der Vorbereitung und Einführung der Europäischen Währungsunion der Wettbewerbsdruck auf die Lohnentwicklung noch einmal verschärft, da nach herrschender Doktrin die Löhne nunmehr das wesentliche, wenn nicht gar einzige verbliebene Instrument zum Ausgleich ökonomischer Ungleichgewichte darstellen sollten. Paradoxerweise wurde zeitgleich mit dem Beschluss zur Einführung der Währungsunion auch die bis heute im EU-Vertrag stehende Regelung eingeführt, wonach Kompetenzen im Bereich der Lohnpolitik für die EU explizit ausgeschlossen werden (Art. 153, Abs. 5 AEUV). Zwar hat diese Regelung die verschiedenen EU-Institutionen (darunter insbesondere die Europäische Kommission und Europäische Zentralbank) nicht davon abgehalten, im Rahmen der allgemeinen wirtschaftspolitischen Empfehlungen massiv für eine wettbewerbsorientierte Lohnpolitik einzutreten. Gleichzeitig wurden jedoch der Möglich keit für alternative Ansätze einer europäischen Lohnkoordinierung auf EU-Ebene von vornherein enge Grenzen gesetzt.

Als Reaktion auf die Euro-Krise hat sich schließlich in jüngster Zeit ein neuer lohnpolitischer Interventionismus herausgebildet, bei dem die EU in bislang unbekanntem Maße direkt Einfluss auf die nationale Lohnpolitik nimmt. Dabei kommt es endgültig zu einem Übergang von einer eher indirekten Lohnkoordinierung durch Wettbewerb und Markt hin zu einer direkten politi schen Koordinierung durch die Institutionen der EU. Der neue lohnpolitische Interventionismus ist dabei Teil eines neuen Systems der europäischen Economic Governance, das neue Regeln und Verfahren hervorgebracht hat, die auf eine wesentlich verbindlichere europäische Koordinierung der Wirtschaftspolitik zielen.

Im Rahmen jährlicher Koordinationsverfahren wie dem sogenannten „Europäischen Semester" und dem Verfahren zur „Vermeidung und Korrektur makroökonomischer Ungleichgewichte" unterbreitet die EU mittlerweile den meisten Mitgliedstaaten mehr oder weniger detaillierte lohnpolitische Vorgaben und Empfeh- lungen, die entsprechend der vorherrschenden Krisendiagnose auf eine Begrenzung oder Senkung der Lohnkosten zielen. Darüber hinaus nimmt die EU erstmals auch die bestehenden Tarifvertragssysteme ins Visier und plädiert in zahlreichen Ländern für eine deutlich stärkere Dezentralisierung der Tarifverhandlungen, die den Unternehmen eine größere (vor allem nach unten gerichtete) Lohnflexibilität ermöglichen soll.

Die entwickelteste Form dieses neuen lohnpolitischen Interventionismus findet sich in den Programmen der sogenannten Troika (Europäische Kommission, Europäische Zentralbank und Internationaler Währungsfond), die Ländern wie z. B. Griechenland oder Portugal für die Zahlung von Krediten aus dem Euro-Rettungsschirm ESM verbindliche politische Auflagen macht. $\mathrm{Zu}$ diesen Auflagen gehören auch umfangreiche Vorgaben zur Entwicklung der Löhne und der Tarifvertragssysteme, die auch vor gezielten und offensichtlichen Eingriffen in die Tarifautonomie nicht zurückschrecken.

Mit der Herausbildung einer expliziten politischen Koordinierung durch die EU, die der Logik einer wettbewerbsorientierten Lohnpolitik folgt, hat sich auch das lohnpolitische Entwick lungsmuster in Europa noch einmal grundlegend verändert. Während bis zum Beginn der EuroKrise in den meisten europäischen Ländern die Reallöhne zwar hinter der Produktivität zurückblieben, die Nominallöhne jedoch in der Regel oberhalb der Preissteigerungsraten lagen, kommt es seither in der Mehrzahl der EU-Staaten zu mehr oder weniger deutlichen Reallohnverlusten Damit wurde de facto eine Lohnsenkungsspirale in Gang gesetzt, die die Nachfrageschwäche in Europa weiter verschärft und die ökonomische Entwicklung insgesamt in eine deutlich deflationäre Richtung treibt.

\section{Perspektiven einer solidarischen Lohn- koordinierung}

Die Alternative zu ökonomischer Stagnation und Krise in Europa liegt in der Entwicklung eines neuen ökonomischen Entwicklungsmodells, das eine einseitige Fixierung auf Wettbewerbsfähig keit und Exportüberschüsse überwindet und deutlich stärker nachfrage- und einkommensgetrieben ist. Hierzu gehört eine Lohnpolitik, die mindestens die Verteilungsspielräume aus nationaler Preis- und Produktivitätsentwicklung ausschöpft und insgesamt auf eine wesentlich egalitärere Einkommensverteilung zielt. Eine solche Lohnpolitik ist jedoch kaum mehr im nati- 
onalen Alleingang, sondern nur im Rahmen einer solidarischen Lohnkoordinierung in Europa möglich.

Erste Ansätze einer solchen solidarischen Lohnkoordinierung lassen sich aufseiten der europäischen Gewerkschaften bis in die 1990er Jahre zurückverfolgen. Im Vorfeld der Europäischen Währungsunion hatten sich diese bereits damals auf europäische Koordinierungsregeln verständigt, die im Wesentlichen den Grundsätzen einer produktivitätsorientierten (Real)Lohnpolitik folgten. Auf diese Weise sollte der europäische Lohnkostenwettbewerb begrenzt und eine weitere Umverteilung zugunsten der Kapitaleinkommen verhindert werden.

Schließlich wurde von den Gewerkschaften auch anerkannt, dass im Rahmen einer Währungsunion der Lohnpolitik eine erhöhte gesamtwirtschaftliche Verantwortung zukommt, vor allem, wenn es darum geht, die Gefahr deflationärer Absenkungswettläufe zu verhindern. Die von vielen Ökonomen empfohlene Unterordnung unter die Zielinflationsrate der EZB wurde von den meisten Gewerkschaften jedoch eher kritisch betrachtet. Zwar kann in einem eher deflationären Umfeld die EZB-Inflationsrate den Gewerkschaften ein zusätzliches Argument für höhere Lohnforderungen bieten. Umgekehrt ist es kaum denkbar, dass bei einer höheren Preisdynamik die Gewerkschaften auf die Sicherung der Reallöhne verzichten, zumal wenn die Preistreiber eher bei externen Faktoren zu suchen sind. In diesem Fall geht es in erster Linie darum, wie die Kosten höherer Preise zwischen Kapital und Arbeit verteilt werden.

Betrachtet man die frühen Ansätze für eine Koordinierung der Lohnpolitik durch die europäischen Gewerkschaften, so muss aus heutiger Sicht konstatiert werden, dass die damals formulierten Ziele nicht erreicht werden konnten. Dies deutet zum einen auf eine gravierende politische Durchsetzungsschwäche vieler europäischer Gewerkschaften hin. Darüber hinaus bestehen ebenfalls große strukturelle Probleme, die in den unterschiedlichen nationalen Tarifvertragssystemen begründet sind, wobei insbesondere der Trend hin zu einer Dezentralisierung der Tarifverhandlungen eine übergreifende Koordinierung erschwert. Der wichtigste Grund dürfte jedoch in der vorherrschenden europäischen Wirtschaftspolitik liegen, die nach wie vor primär darauf ausgerichtet ist, durch Liberalisierung und Deregulierung den europäischen Wettbewerb zu intensivieren und Arbeitnehmer- und Gewerkschaftsrechte zu schwächen. Angesichts eines solchen entgegenlaufenden politischen Umfeldes kann von den Gewerkschaften kaum erwartet werden, dass sie quasi isoliert von sonstigen Entwicklungen eine solidarische Lohnkoordinierung durchsetzen.

Die Umsetzung einer solidarischen Lohnkoordinierung als politisches Gegenprojekt zum gegenwärtig dominierenden lohnpolitischen Interventionismus in Europa ist demnach höchst voraussetzungsvoll. Sie bedarf einerseits einer organisatorischen und machtpolitischen Stärkung der Gewerkschaften sowohl auf nationaler als auch auf europäischer Ebene. Darüber hinaus bedarf sie aber auch einer aktiven Unterstützung durch die europäische Politik. Damit ist nicht gemeint, dass etwa die EU-Kommission oder der Europäische Rat zukünftig Vorgaben zur allgemeinen Lohnentwicklung machen sollen. Es geht vielmehr um flankierende politische Projekte, die einer autonomen europäischen Lohnkoordinierung durch die Gewerkschaften mehr Durchschlagskraft verleihen können.

Angesichts der aktuellen Entwicklung in Deutschland, wo mit der Einführung eines gesetzlichen Mindestlohns und der Stärkung des Tarifvertragssystems durch eine Reform der Allgemeinverbindlicherklärung eine neue lohnpolitische Richtung eingeschlagen wird, könnten auch auf europäischer Ebene zwei zentrale Ansatzpunkte formuliert werden: Zum einen könnten die Vorschläge für eine europäische Mindestlohnpolitik aufgegriffen werden, bei denen es darum geht, europaweite Normen für ein angemessenes Mindestlohnniveau (z. B. 60 \% des nationalen Medianlohns) festzulegen. Durch eine europäische Absicherung des Lohngefüges nach unten könnte die Lohnentwicklung in Europa insgesamt stabilisiert und der Druck auf die Tarifpolitik gemildert werden. Zum anderen könnte die EU, statt ihre Energie auf die Dezentralisierung und in vielen Fällen faktische Zerstörung von Tarifvertragsstrukturen zu richten, eine europaweite Initiative zur Stärkung der Tarifautonomie durchführen. Hierzu gehören der Ausbau überbetrieblicher Tarifvertragssysteme und die Erhöhung der Tarifbindung, die durch die Stärkung von Gewerkschaftsrechten und politische Stützungsinstrumente (wie z. B. die Allgemeinverbindlicherklärung) gefördert werden können.

Schließlich muss neben eine europäische Mindestlohnpolitik und eine europäische Initiative zur Stärkung der Tarifautonomie auch eine andere europäische Wirtschaftspolitik treten. Diese muss darauf ausgerichtet sein, eine Überforderung der Lohnpolitik beim Ausgleich ökonomischer Ungleichgewichte zu vermeiden und eine solidarische Lohnkoordinierung in eine umfassende Koordinierung der gesamten Wirtschaftspolitik einzubetten.

\section{AUTOR}

THORSTEN SCHULTEN, Dr., ist Wissenschaftler im Wirtschafts- und Sozialwissenschaftlichen Institut (WSI) in der Hans-Böckler-Stiftung. Arbeitsschwerpunkte: Arbeits- und Tarifpolitik in Europa.

thorsten-schulten@boeckler.de 